\title{
DIFERENTES CAMINHOS DE SOCIALIZAÇÃO ATÉ A ADOLESCÊNCIA
}

\author{
DIFFERENT SOCIALIZATION PATHWAYS \\ TO ADOLESCENCE
}

Heidi Keller $^{1}$

KELLER, K. Diferentes Caminhos de Socialização até a Adolescência. Rev. Bras. Cresc. Desenv. Hum., SãoPaulo, 8(1/2), 1998.

Resumo: A diferenciação do curso da vida humana em fases pode ser encarada como uma adaptação a demandas contextuais. As abordagens sociobiológicas têm atribuido às fases desenvolvimentais o status de conquistas filogenéticas ao invés de estágios transitários. Da mesma forma, o padrão e a duração das fases diferem através de contextos e culturas. A infancia é vista como a fase em que uma matriz social primária é construída. Embora esta tarefa desenvolvirnental seja universal, sua elaboração especifica difere em backgrounds culturais distintos. Os resultados desenvolvimentais desta fase fornecem as bases para modos especificas de exploração e aprendizado durante a infancia - a fase de aquisição. Uma trajetória tipicamente ocidental e uma trajetória tipicamente não-ociãental são apresentadas, levando, em seguida, a diferentes concepções de adolescência: de um lado, como uma moratória educacional e de desenvolvimento do sela; e, do outro, como o inicio da vida reprodutiva do adulto.

Palavras-chave: pesquisa de história de vida; contextos de socialização; individualismo.

\section{INTRODUÇÃO}

A psicologia evolucionária moderna propõe um padrão de curso de vida típico à espécie. Como K-estrategistas ( $\mathrm{K}=$ detetor de densidade populacional em termos de pesquisa populacional, PIANSKA, 1970), os humanos têm os períodos mais longos de infância e de juventude (cf. fig. I), acompanhados de cuidados maternos e paternos intensivos, sendo únicos no mundo animal.

O cuidado parental, nesta teoria, é concebido como um investimento que os pais alocam à sua prole baseado em cálculos custo-beneficio individuais e, principalmente, implícitos, que objetivam otimizar o sucesso reprodutivo (genético). O sexo e a posição ordinal dos descendentes foram especialmente identificados como dirigindo os investimentos parentais, contingente aos recursos disponíveis. "Contabilidades” sociobiológicas concebem o investimento parental principalmente como uma dimensão quantitativa onde "mais" ou "menos" pode ser distribuído. "Mais ou menos", em termos de cuidado ou negligência, igualmente é identificado, em humanos, como uma importante condição que define o contexto infantil. Circunstâncias aversivas, paticularmente,

1 Professor na Universidade de Onsbruck, Alemanha. O autor agradece a Jovsi Relinidis Dzeaye pelos vários insights às culturas de NSO e Fulbe em Camarões. Texto lido na $4^{\mathrm{a}}$ África Region International Society of Behavioral Development Conference (ISSBD), Windhock/Namibia, de 20 a 23 de julho de 1998.

Tradução: Elaine Pedreira Rabinovich. 
influenciam o timing de marcadores biológicos, como o início da puberdade (BELSKY et al., 1991; CHISHOLM, 1993; CHASIOTIS, 1998). As expenências na infância, por sua vez, induzem estratégias reprodutivas, que são concebidas como quantitativas (muitos filhos, baixo investimento parental) ou qualitiva (poucos filhos, alto investimento parental).

Há, contudo, diferentes qualidades de cuidados parentais prevalecentes nos humanos, es-

Figura 1 - O desenvolvimento do ciclo vital em primatas (JOLLY, 1975)

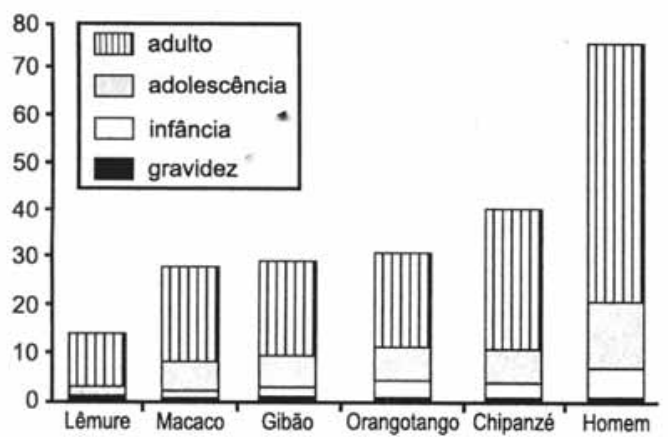

pecialmente quando se compara diferentes culturas, que não podem ser capturadas apenas por diferenças quantitativas (KELLER, 1998, in press). Modos diferentes (A e B) de investimento parental são sumariados no quadro 1 , diferenciando cuidado físico e emocional. É óbvio que os dois modos não podem ser comparados a respeito de "mais ou menos" investimento, mas representam diferentes estratos de socialização.

Como proposto em modelos de socialização sociobiologicamente orientados, a relação entre o contexto infantil e as estratégias reprodutivas é mediada pela qualidade do apego (BELSKY et al., 1991; CHISHOLM, 1993). Por meio disto, representações de relações em termos de estruturas de personalidade são introduzidas como uma interface entre demandas contextuais e metas reprodutivas. A evolução da personalidade tem sido equivocadamente ligada à regulação social necessária a vida em grupo da espécie (Mac DONALD, 1988). Em conjunto com a natureza basicamente "egoísta” em perseguir seus intereses genéticos, a personalidade humana pode ser descrita como um equilíbrio especial entre centração no ego, de um lado, e centração na relação, do outro. Sem entrar em uma detalhada discussão de concepções como a de individualismo/coletivismo (KAGITCIBASI, 1997) ou construções inde-

Quadro 1 - Dois tipos de investimento parental

\begin{tabular}{|l|l|l|}
\hline \multicolumn{1}{|c|}{ Cuidado físico } & \multicolumn{1}{|c|}{ A } & \multicolumn{1}{c|}{ B } \\
\hline \multicolumn{1}{|c|}{ Aleitamento } & \multicolumn{1}{|c|}{ 2-4 anos } & \multicolumn{1}{c|}{ 1-3 meses } \\
\hline Carregar & $\begin{array}{l}\text {-mais de 50\% do dia durante os primeiros } \\
\text { anos (Aka Pygmy: HEWLETT, 1991; Efe: } \\
\text { TRONICK } \text { et al., 1992) }\end{array}$ & $\begin{array}{l}\text {-principalmente como uma } \\
\text { curta resposta ao choro }\end{array}$ \\
Contato corporal & $\begin{array}{l}\text {-dia e noite ("black and hip culture", } \\
\text { WHITING, 1981; co-sleeping) }\end{array}$ & $\begin{array}{l}\text {-pouco: bebês "empacotados" } \\
\text { (WHITING, 1990; culturas do } \\
\text { berço, WHITING, 1981) }\end{array}$ \\
Cuidado extra maternal & $\begin{array}{l}\text {-outros parentes; irmãos } \\
\text {-longuda paga }\end{array}$ \\
& $\begin{array}{l}\text {-contato e comunicação corporais } \\
\text { prolongados } \\
\text {-apoio a uma longa simbiose }\end{array}$ & $\begin{array}{l}\text {-curtos períodos de cuidado } \\
\text { exclusivo } \\
\text {-breves episódios face-a-face } \\
\text {-incentivo à independência } \\
\text { precoce }\end{array}$ \\
\hline
\end{tabular}

Ver KELLER, 1998, in press.

pendente ou interdependente do self (MARKUS \& KITAYAMA,1991,1998; para uma discussão, KELLER, 1998, in press; KELLER \& ECKENSBERGER, 1998), pode ser dito, contudo, que diferenças culturais quanto às concepções de self ou de personalidade podem ser localizadas nas dimensões relacional e/ou independência. Consequentemente, diferenças em contextos de criação e de cuidados parentais foram relacionadas a diferentes metas de socialização (LeVINE, 1990; 
KELLER et al., 1997). KELLER \& ECKENSBERGER (1998) formularam diferentes caminhos de socialização da infância à adolescência que podem ser ligados ao desenvolvimento de diferentes concepções do self. Estes caminhos serão introduzidos nas próximas seções.

\section{DOIS CAMINHOS DESENVOLVIMENTAIS ${ }^{2,3}$}

O conceito de desenvolvimento aqui apresentado enfatiza a aquisição ativa/construtiva da informação ambiental organizada através de tarefas desenvolvimentais para as quais o indivíduo está biologicarnente predisposto. KELLER \& ECKENSBERGER (1998) propuserarn a repartição do curso de desenvolvimento do nascimento à fase adulta em três fases funcionais:

1. fase de recepção ${ }^{4}(0-2 / 3$ anos);

2. fase de aquisição (fim da fase de recepção até a entrada na puberdade);

3. fase de transformação (entrada na puberdade até a reprodução).

Os rótulos das fases caracterizam os mecanismos desenvolvimentais dominantes em cada uma delas, sem negar que tanto outros mecanismos são efetivos em cada fase quanto ocorre a presença de mecanismos anteriores em fases posteriores. A duração das fases e a transição de fase a fase são culturalmente variáveis. O limite das fases é definido por marcadores psicobiológicos. A idade de 2 a 3 anos é obviamente encarada na maioria das culturas como uma transição desenvolvimental, dado que naquela idade campos sociais além do grupo social primário são abertos. Nesta idade, as crianças alemãs entram no Jardim da Infancia; na Índia, "chuakarma" indica que a criança está agora pronta para o processo de disciplinização (SARASWATHI \& PAI, 1997); a criança NSO está pronta para deixar a fazenda familiar e receber ensinamentos de adultos sem considerar o grupo de origem. A preocupação com o cuidado do bebê cede a vez para a preocupação com a formação correta do caráter como um mem- bro da comunidade (DZEAYE, in prep., MZEKA, 1996). A entrada na puberdade e o início da reprodução são biologicamente definidos, mas as transições desenvolvimentais são interpretadas diferentemente segundo as culturas. As fases são consideradas como caracterizando padrões de desenvolvimento prototípicos (protótipo sensu BISCHOF, 1996, p. 684f) que definem um enquadramento desenvolvimental em uma orientação individual.

Parece surpreendente que embora haja um número infinito de práticas culturais e variações em estilos de vida, dois padrões consistentes podem ser diferenciados através das culturas que são usualmente denominadas, na nossa linguagem prevalecente etnocêntrica, como Ocidental (por exemplo, Europa e Norte América que representam cerca de $14 \%$ da população mundial) ${ }^{5}$ e nãoOcidentais (representando o resto do mundo com cerca de $86 \%$ da população mundial). Estes dois padrões podem ser compreendidos como expressando unidades básicas de uma arquitetura psicológica universal (KELLER et al., in prep. a) que são combinados em misturas específicas que então formam os protótipos culturais. Caminhos individuais podem divergir dos protótipos culturais dependendo, por exemplo, da estratégia sociocultural que deve ser atingida.

\section{A Fase de Recepção}

Devido à natureza social dos humanos pode ser assumido que a primeira tarefa desenvolvimental consiste em adquirir uma matriz social básica (primária) que deve ser adaptativa ao respectivo ambiente ${ }^{6}$. A altricialidade das crianças e a concepção compensatória do investimento parental implica que estas relações são assimétricas no sentido que "... os pais tomam a direção..." (WALDEN, 1996, p.2074). As crianças crescem usualmente dentro dos scripts de seus pais que projetam os cenários (settings) para sua prole e atribuem as atividades diárias apropriadas, os arranjos de dormir, os contatos sociais, e os cuidados parentais. Estas atividades cultura-específicas podem ser especificadas em cenários que

2 Referimo-nos ao ciclo vital humano como começando com o nascimento, embora estejamos conscientes de diferentes concepções culturais, por exemplo, que integram a fase pré-natal (NSAMENANG, 1992; DZEAYE, in prep.)

3 A discussão a seguir está baseada em KELLER \& ECKENSBERGER (1998).

4 O termo "recepção" não exclui a participação ativa das crianças nos processos socializatórios.

5 Esses números estão publicados no World Almanac of Facts, apud RUDMIN 1996).

6 O desenvolvimento do apego, que atraiu substancial atenção nos estudos inter-culturais (COLE, 1992), não será considerado aqui, desde que ele representa uma aplicação culturalmente insensível de procedimentos de avaliação que foram desenvolvidos como uma adaptação culturalmente informada, assim colocando tremendos problemas conceituais e teóricos. Também a suposição de que as distribuições normativas ocidentais representam normas desenvolvimentais universais (SROUFE \& WATERS, 1997) é avaliada criticamente. 
proporcionam contextos interacionais ${ }^{7}$. Cenários definem condições ambientais concretas para as experiências iniciais de socialização.

KELLER \& ECKENSBERGER (1998) propuseram classificar os cenários de acordo com dois critérios: o meio social e a estruture atencional. O modelo ocidental de interação precoce paicriança concebe o cuidado come uma troca comportamental exclusiva. Mais prevalecente na população mundial é outra concepção, especialmente sob uma condição da escassez de recursos, onde o cuidado infantil compreendido como uma atividade co-ocorrente (SARASWATHI, 1994), deste modo descrevendo a socialização como um processe co-ativo. $\mathrm{O}$ foco atencional no cuidado ocidental está exclusivamente sobre a criança; já o cuidado co-ativo implica em uma distribuição da atenção a diferentes focos ao mesmo tempo.

Embora as diferenças nos cenários de cuidados infantis através das culturas sejam substanciais é, contudo, óbvio que nas mais divergentes culturas são as mães as pessoas primárias socialmente significantes durante os meses inicias da vida $^{8}$. Há, entretanto, consideráveis diferenças através das culturas com respeito a oportunidades e ocorrências de interações com outros, principalmente com outros membros da família. Na família ocidental (nuclear), as crianças gastam a maior parte do dia sozinhas com suas mães (MUNROE \& MUNROE, 1994; 20 a 25\% do dia) ou mesmo completamente só (1/3 do seu tempo WHITING, 1981). Nas culturas não-ocidentais mãe e criança gastam quase todo o dia juntas com outros membros da família ou da residência ou estão encaixadas na vida comunitária dos vilarejos (TRONICK et al., 1992: Efe; EIBLEIBESFELDT, 1984: Himba; RABINOVICH Brasil). ${ }^{9}$ Crianças no território de Mbven, nos Carnarões, são deixadas sozinhas no máximo duas horas ao todo durante o dia inteiro, quando estão dormindo (DZEAYE, in prep.).

Contextos de cuidados, portanto, podem ser qualificados como múltiplos quando diferentes pessoas estão disponíveis como parceiros interacionais. Estes contextos múltiplos são diferenciados dos diádicos onde mãe e criança estão cozinhas. A definição de cenários sociais está apresentado no Quadro 2.
Quadro 2 - Definição de cenário (setting) social

\begin{tabular}{|c|c|c|c|}
\hline & \multicolumn{2}{|c|}{ Estrutura atencional } \\
\hline & & co-ativa & exclusiva \\
\hline meio social & $\begin{array}{c}\text { diático } \\
\text { múltiplo }\end{array}$ & não-Ocidental & Ocidental \\
\hline
\end{tabular}

In: Keller \& Eckensberger, 1998, p. 72.

A maioria dos estudos sobre sacializacão precoce está obviamente localizado no contexto diádico/exclusivo que, ao mesmo tempo, constitui o menos presente no mundo.

Igualmente, as experiências concretas interacionais variam segundo os cenários. Um modo distal de interação focalizando sobretudo a comunicação visual e a troca vocal/verbal parece ser especialmente prevalecente nos cenários diádicos exclusivos. Uma estrutura de diálogo préverbal inicial com episódios turn-taking é especialmente criada por respostas parentais contingentes em direção aos sinais da criança, parecendo ser agradável a ponto de ser mantida para ambos, pai (usualmente mãe) e criança. Nos cenários de cuidado co-ativo, o modo prevalente de interação parece ser o contato corporal ("back and hip cultures" versus "crib and cradle culturas", WHITING, 1990); por exemplo, bebês norte-americanos são carregados apenas metade do tempo que os bebês Gusii são carregados (WHITING, 1981). Carregar e contato corporal, assim, provem a criança com estimulação tátil e vestibular que cria a experiência interacional de valorosidade emocional (KELLER \& ECKENSBERGER, 1998; KELLER et al., in prep. b). Através da análise interacional extensiva de situações de interação precoce mãe-criança pudemos demonstrar que a experiência de contingência e calorosidade formam dois componentes parentais separados (KELLER, VOELKER \& ZACH, 1997; KELLER et al., in prep b; VOELKER et al., in press). Os dois componentes proporcionam à criança diferentes experiências interacionais e, assim, diferentes informações sobre o self e a relação.

Sobretudo a contingência parental em termos de reatividade rápida em direção aos sinais

7 Esta abordagem é considerada pennitir mais especificações comportamentais do que o conceito de nicho de desenvolvimento (SUPER \& HARKNESS, 1996).

8 Mesmo em contextos de mútiplo cuidado, as mães gastam ^sO \% do dia com seus filhos, como TRONICK et al. (1992 formularam para os Efe no Zaire. Existem, contudo, também culturas onde as crianças são vistas como propriedade d. comunidade (por exemplo, os NSO,DZEAYE, in prep.).

9 É óbvio que não são primariamente destinatárias desta concepção as populações urbanas em culturas não-ocidentais. 
infantis (período de latência abaixo de 1 segundo) favorece as experiências de causalidade, preditibilidade, controlabilidade. O contexto interacional do contato corporal porporciona à criança a experiência de harmonização calorosa e positiva. A calorosidade pode ser vista favorecendo sentimentos de relação, empatia e condescendência (KELLER et al., in prep b). Os componentes interacionais de contingência e calorosidade formam as propensões parentais para a motivação infantil para experienciar calor (HARLOW \& HARLOW, 1962) e para detectar contingências (TARABULSY et al., 1996). Eles podem ser concebidos como aspectos universais de comportamentos parentais que ocorrem em diferentes misturas em diferentes contextos e, assim, variam não apenas inter-culturalmente mas também interindividualmente.

Diferentes metas de socialização como expressas em etnoteorias parentais e concepções implícitas de cuidados parentais e de desenvolvimento (KELLER, 1997; KELLER \& ECKENSBERGER, 1998) espelham essas diferenciações comportamentais. Pode ser sumarizado que as culturas ocidentais promovem a meta de socialização de autonomia precoce com o bebê passando uma quantidade substancial do tempo sozinho, sendo a estrutura interacional inicial diádica-distal e exclusiva, dirigida para controlar o ambiente (preditibilidade, segurança). A regulação interna dos afetos é imposta. Exceto a mãe como cuidador primário, há poucos outros parceiros sociais. O interesse no ambiente é precocemente estimulado. $\mathrm{O}$ auto-conceito respectivo pode ser conceitualizado como "self como agente" com controle acional da ação.

Em muitas culturas não-ocidentais, promove-se a meta da socialização de interpelações sociais próximas entre cuidadores e criança: os bebês passam a maior parte do seu tempo com diferentes pessoas (cuidado múltiplo) onde a díade mãe-criança está embebida; íntimo contato corporal e frequente acalanto (nursing) apoiam uma dependência corporalmente baseada com os cuidadores primários, induzindo a unidade emocional (simbiose) e relacional; o cuidado é co-ativo (atividade “co-occurente”). O conceito imposto de self pode ser encarado como "self como coagente”, com controle empático da ação.

Durante a recepção inicial do respectivo contexto cultura-específico, padrões básicos e orientações sociais são adquiridos com consequências para o curso desenvolvimental posterior.
A evidência empírica refere-se, e praticamente dirige-se, aos meses iniciais de vida. Em muitas culturas, uma primeira transição desenvolvimental é definida em torno do terceiro mes de vida. Na literatura ocidental, esta transição é interpretada como uma "mudança biocomportamental" (EMDE, 1984; COLE, 1992), como o real início de orientação social (sorriso social: BAYLEY, 1955), ou como um primeiro resultado desenvolvimental a respeito da formação de relação (KELLER, 1997). Em culturas não-ocidentais, transições cerimoniais podem ser observadas durante esta mesma época, como, por exemplo, a cerimônia de atribuição de nome na Índia (nalamakarma), onde a criança é introduzida no mundo ao ser exposta ao sol ou à lua pela primeira vez (nirhakarma) SARASWATHI \& PAI, 1997, E 75). É interessante notar que os processo maturacionalmente definidos (por exemplo aquisição do ritmo circadiano), que principalmente definem a mudança biocomportamental, não ocorrem no contexto hindu. Estes exemplos apoiam fortemente a visão de que as transições precoces desenvolvimentais já espelham os conceitos culturais.

Até o fim da fase de recepção, com cerca de 2 a 3 anos, os padrões adquiridos sociais se estabilizam e preparam o caminho para novos desafios desenvolvimentais, especialrnente pela abertura de novos contextos. A criança Zinacanteca tanto quanto a criança do vilarejo indonésio são carregadas até a idade de dois anos (GREENFIELD \& CHILDS, 1991) e então começam a se movimentar independentemente; a criança alemã entra no Jardim de Infância com cerca de 3 anos e se torna um membro do grupo de co-etanos; supõe-se que criança hindu tenha completado o processo de individuação ${ }^{10} \mathrm{com}$ cerca de 3 anos de vida (não se pretendendo que a relação próxima com c pais seja dissolvida: SARASWATHI \& PA 1997). Em muitas “culturas orgânicas (SHWEDER \& BOURNE, 1982) as criança são desmamadas com cerca de três anos de idade (SHOSTAK, 1981). Com estas transiçõe a criança se torna pronta para a sua introdução em novos contextos de aprendizagem.

\section{A Fase de Aquisição}

Foi dito que, durante a fase de recepção, a organização básica da personalidade em termos de conceitos de self e relação, foi adquirida. Contingente a estas estruturas, os indivíduos desenvolvem domínio e competência em suas respectivas culturas. As estruturas cognitivas de aquisição

10 Isto sublinha a visão de que os conceitos de autonomia vs. dependência não capturam diferenças culturais. 
e processamento de informação, como percepção, memória, resolução de problema e criatividade são, particularmente, os mecanismos pelos quais os indivíduos se tornam aptos a realizar os papéis adultos. As culturas, contudo, diferem substancialmente no que elas entendem como inteligência. O Ocidente enfatiza o pensamento abstrato, lógico como a meta desenvolvimental, por exemplo, expresso na concepção de inteligência de PIAGET ( 1934) enquanto conceitos não-ocidentais, por exemplo, o " $n$ 'glouele ${ }^{11}$ Baoulé da Costa de Marfim (DASEN et al., 1985) enfatiza "componentes tecnológicos” como observações, habilidades, atenção, aprendizagem rápida, boa memória, conhecimento escolar, mas também aspectos práticos como destreza manual, escrita e desenho e, acima de tudo, competências sociais extensivas a categorias éticas e morais. Dever e responsabilidade, iniciativa e honestidade, respeito, obediência, polidez, mas também contar histórias e a capacidade de falar de uma maneira socialmente apropriada são componentes do conceito de "n'glouele". Harmonia social é também parte da inteligência Cree no Alasca (BERRY \& BENNETT, 1992) ou dos A-Chewa na Zambia (SERPELL, 1977). SERPELL ( 1984, p. 1 19) sumariza os estudos africanos com diferenças reconhecíveis: “...Pequenas comunidades agrárias na África avaliam a disposição social-cooperativa obviamente como um componente integral da inteligência. A vivacidade cognitiva que não é usada de um modo social-construtivo é encarada como uma coisa perigosa".

Com essas diversas concepções de inteligência e competência, também os processos de aquisição em termos de contextos de aprendizagem diferem inter-culturalmente. Nas culturas ocidentais, assim como nas classes sociais média e média-superior de culturas não-ocidentais, as crianças aprendem predominantemente em contextos especificamente definidos. Em instituições escolares, a educação formal é adquirida através de técnicas culturais de aprendizagem como leitura, escrita e matemática, sem levar em conta a sua aplicação. Em muitas culturas não-ocidentais, contudo, a própria cultura é o conteúdo do desenvolvimento (GREENFIELD, 1996), por exemplo, as técnicas culturais são adquiridas dentro do trabalho e dos contextos de vida específico.

Contextos de aprendizado diário podem ser concebidos como "participação guiada e apropriação de atividades sócio-culturais da criança"
(ROGOFF, 1990). ROGOFF ei al. ( 1993) conduziram um estudo inter-cultural comparativo com uma amostra de uma cidade indígena na Guatemala, uma amostra de classe média de uma cidade norte-americana, uma amostra da classe média de uma cidade da Turquia e uma amostra de uma vila tribal na Índia”. No caso das amostras de classe média, os adultos definem o contexto de aprendizado para as crianças e focalizam sobre o modo de comportamento verbal e instruções explícitas; nas comunidades das vilas, as crianças tinham responsabilidades pelos seus próprios processos de aprendizagem observando e participando nas atividades dos adultos com um claro foco em comportamento nãoverbal. Este estudo, além disto, revela uma diferença interessante na distribuição da atenção dos cuidadores. Nas amostras urbanas de classe média, a motivação do ensino dos cuidadores estava presente somente na tarefa explícita de ensino (explicando um novo brinquedo), enquanto as famílias rurais permaneciam sensíveis a suas crianças durante todos os tipos de tarefas. Nas amostras de classe média, os processos atencionais eram alternativos e exclusivos, enquanto nas comunidades dos vilarejos mãe e criança estavam constantemente atentas à outra, mesmo quando elas estavam envolvidas em diferentes atividades. O cuidado coocorrente mudou, deste modo, para a atenção coocorrente dos cuidadores, e uma distribuição atencional coocorrente já foi adquirida pelas crianças. Também GREENFIELD (1996; GREENF1:ELD \& CHILDS, 1977) confirmaram a importância especial do aprendizado observacional para a tecelagem em índios Zinacantecanos no México. Ela fez uma importante observação posterior introduzindo o conceito de conhecimento compartilhado. As meninas que aprendiam a tecer padrões complicados, culturalmente prescritos, não deveriam responder as perguntas ou dar informações sobre o que faziam, dado que suas mães tinham mais conhecimento. Respondendo as questões, as meninas demonstrariam um ponto de vista independente em termos de conhecimento independente. Era considerado mais adequado que as andes mais experientes ou a família como um todo respondessem às questões, dado que uma maior precisão na informação requisitada poderia ser adquirida através da união de esforços. O conhecimento, portanto, é considerado como representando uma propriedade "partilhada" de uma família. Também nas famílias artesãs de índios norte-americanos, o conceito de competência interpessoal é encarado

11 Embora estejamos nos referindo as crianças além dos dois a três anos em sua fase de aquisição, as crianças (toddlers) nestas amostras tinham somente 12 a 24 meses; os mecanismos observados são, todavia, válidos também para crianças mais velhas. 
como a capacidade de cooperação com outros de modo a alcançar as metas grupais. Isto é mais levado em consideração do que a competência individual e a responsabilidade individual para a realização (ANADAL AKSHMY, 1975). Igualmente em culturas africanas (por exemplo, Senegal: RABAIN-JAMAIN, 1979; Quênia: HARKNESS \& SUPER, 1977) as crianças são encorajadas a resolver problemas diários como um grupo (SERPELL, 1984).

Com esta última observação, as metas desenvolvimentais da fase de aquisição estão já indicadas. Embora hipotético e especulativo, podemos especificar os contextos de aprendizagem culturaespecíficos para diferentes metas desenvolvimentais. Argumentamos que nas culturas ocidentais (e na classe média e média-superior de várias das culturas não-ocidentais), o conhecimento é adquirido como uma "posse" descontextua-lizada individual e privada que é aprendida através de instruções formais, e especialmente verbais, por pessoas especificamente treinadas em contextos especificamente definidos que estão segregados do mundo adulto. $\mathrm{O}$ foco atencional é específico e exclusivo. O conhecimento adquirido deve ser individualmente administrado. Em muitas culturas não-ocidentais, particularmente em estruturas rurais de artesãos, o aprendizado é focalizado sobre observações auto-responsáveis e participação prática em atividades constituintes de conhecimento, com múltiplas orientações atencionais. O conhecimento resultante contribui ou amplia as competências da família como um todo onde o papel de conformidade é especialmente valorizado.

A orientação para estas diferentes metas desenvolvimentais têm implicações para as diferenciações de construções psicológicas, como por exemplo a construção do self. MARKUS \& KITAYAMA (1991) sumarizam um conjunto de estudos demonstrando que o orgulho é uma emoção que pode ser identificada mais facilmente em pessoas com um self independente, enquanto a vergonha pode ser relacionada a personalidades com um self interdependente. O modo ocidental de feedback contínuo, principalmente em termos de avaliações, poderia ser contrastado, por exemplo, com a atitude indígena do impacto educacional sobre corrigir ou disciplinar (ANANDALAKSHMY, 1991; DAVE, com. pes.).

Enquanto culturas promovendo individualidades necessariamente enfatizam a variabilidade interindividual, é a pressão para conformar (ou melhor, empenhar-se por harmonia), do outro lado, que não tolera o desvio individual. Pertencer a um grupo com uma estrutura de papel ou de hierarquia explícita é oposto ao modo ocidental de múltiplas associações de grupo (trabalho, fa- mília, amigos, sindicatos etc.) com direitos igualitários, pelo menos teoricamente formulados.

A base que foi colocada na fase de recepção, com a construção de uma orientação social básica, recebe o imprinting cultural concreto durante a fase de aquisição. O curso desenvolvimental da fase de aquisição depende da definição de adolescentes como uma moratória juvenil ou como o começo da fase adulta com a fundação da família e com responsabilidades financeiras.

\section{Fase de Transformação}

O principal objetivo da fase de transformação é a preparação cultural para a definição social do adulto. Enquanto a fase de recepção não varia com respeito à sua duração em diferentes culturas e, igualmente, a fase de aquisição cobre um segmento desenvolvimental comparável, a duração da fase de transformação representa um fenômeno especifico significativo à cultura. Sob condições econômicas restritas, o nivel educacional que é alcançado ao final da fase de aquisição (entrada na puberdade) é visto como suficiente para poder contribuir com uma completa capacidade econômica para a família. Sob circunstâncias econômicas afluentes, uma fase especial adolescente é definida como um estágio acessório de vida em termos de uma moratória educacional, onde também a formação de identidade é postergada. SARASWATHI \& PAI (1997) reivindicam igualmente que, para níveis sociais baixos ou pessoas rurais na Índia, a fase de "brahmachrya" (o tempo de aprendizado que é equivalente à adolescência ocidental onde a competência e consciência dos deveres são adquiridos) é mais curto do que em cenários urbanos, especialmente nas classes média e superior.

O segundo marcador com respeito à definição de adulto refere-se ao início da reprodução e, respectivamente, do casamento. A idade à primeira progenitura define o fim da fase de transformação através das culturas e o status de adulto (por exemplo, NSO nos Camarões, NSAME-NANG, com. pes.; DZEAYE, in prep.). Em algumas culturas, é mesmo especificado que somente o nascimento do primeiro filho define a fase adulta (LLOBERY, 1978); em NSO, um homem que morre solteiro e sem filhos é enterrado sem qualquer cerimónia, porque ele é ainda uma criança (DZEAYE, in prep.). A idade da primeira progenitura é mais ou menos contingente à entrada na puberdade em algumas culturas principalmente não-ocidentais, enquanto representa um evento auto-selecionado em outras, principalmente em culturas ocidentais, com primiparas de 40 anos ou mesmo mais velhas não sendo exceção. 
Embora rituais de transição ("ritos de passagem”, van GENNEP, 1960) existam também nas culturas ocidentais (por exemplo, a confirmação para cristãos protestantes ou rituais específicos de clubes da juventude em culturas ocidentais), eles representam iniciações prescritas socialmente baseadas principalmente em culturas onde a transição da infância para o adulto é mais ou menos direta. LLOBERY (1978, p. 100f ) por exemplo, descreve a cerimónia de iniciação do menino Ndembu na Zâmbia como uma festividade de quatro meses, onde os meninos são separados da tribo e introduzidos nos papéis adultos. A iniciação no meio africano constitui o que MVENG (1974) chama "uma escola fundamental" onde os homens jovens são submetidos a todos os tipos de testes: resistência física realizada fora da vida do vilarejo e com ausência de contato feminino.

MacDONALD (1988) propõe encarar a produtividade econômica como a chave para compreender a variabilidade das práticas rituais culturais. Sociedades com relativamente baixo nível de produção realizam rituais reprodutivos sucintos para meninas a fim de encontrar pares atraentes ou parceiros de casamento. Em sociedades economicamente mais prósperas, os sistemas familiares extensos ou conjuntos (que controlam os recursos) garantem o casamento adequado das filhas, também sem rituais especiais, de modo que eventualmente mesmo um “dote de noivado” pode ser pedido.

Durante os períodos de iniciação à adolescência ocidental tanto quanto a não-ocidental, o grupo de coetâneos tem um papel especial em se tornar uma força socializadora maior. As culturas diferem, contudo, com respeito à definição do grupo de colegas (peer), com as culturas ocidentais baseadas principalmente em amizade devido às orientações de valores e interesses partilhados; árabes israelitas, por exemplo, encaram sua rede de linhagem, de primos primeiros, segundos e terceiros como seu grupo de colegas (peer) (SEGI-NER, 1995). EL-SHAMY (1981) relatou sobre grupos de colegas diferentemente íntimos no mundo árabe: o mais intimo é constituído por irmãos, seguido pelo grupo de vizinhos e então colegas de escola e outros parentes. Em culturas altamente segregadas quanto ao sexo, os caminhos desenvolvimentais de meninas e meninos são mantidos separados o mais tardiamente possível. Em NSO, os jovens homens têm que adquirir tarefas desenvolvimentais que estão relacionadas a responsabilidades sociais e financeiras, enquanto as mulheres jovens têm de documentar sua prontidão para o casamento e para a capacidade de procriação (DZEAYE, in prep.).
Como foi dito, a definição e duração da fase de transformação formam a base para a extensão dos desvios tolerados do protótipo cultural. Nas culturas ocidentais, é esperado que o adolescente construa e co-construa sua própria identidade transformando os padrões culturais do coletivo em um sistema individual de valores e crenças. Com estas concepções individuais do self; as diferenças entre indivíduos aumenta. Se, ao contrário, a identidade é definida como uma contribuição ao grupo social, a aquisição de padrões culturais tem de ser mais replicativa, e assim, confinar a definição de cultura pessoal. Isto implica em uma transição mais ou menos direta e imutável da cultura coletiva de geração a geração. Contudo, os mecanismos de transferência têm de ser mais elaborados. Parece haver evidência de que o calor emocional que é experienciado dentro do contexto familiar, e especialmente nas relações pai-criança, como foi descrito anteriormente, influencia a aceitação de valores e atitudes parentais.

"Interações pai-criança afetivamente quentes tenderiam a facilitar a adoção de atitudes e valores parentais e resultam em um mecanismo conservador para a transmissão da cultura." (Mac DONALD, 1988, p. 161; cf. também BANDURA \& HUSTON, 1961).

MacDONALD (1988), contudo, restringe esta visão a sistemas de famílias nucleares, ao passo que considera o controle parental como garantindo conformidade com valores parentais em sistemas de família polígonos (por exemplo, os Gusii). Apenas estudos empíricos podem ajudar uma melhor compreensão destes mecanismos de transmissão.

A definição social de adulto canaliza a respectiva diferenciação do papel societal. Enquanto em culturas ocidentais o papel e o status são primariamente definidos por impedimentos econômicos que estão, ao menos teórica e legalmente, consolidados como reivindicação por iguais oportunidades, em muitas culturas não-ocidentais a idade e o sexo são os parâmetros que definem as estruturas hierárquicas dos sistemas sociais. Os subsequentes diferentes direitos e deveres estão frequentemente ancorados em estruturas de significado metafísico. "Ter filhos homens é o maior dever de uma pessoa ... ele reduz a dívida com relação aos ancestrais”" (SARASWATHI \& PAI, 1997, p. 82). Seguindo a proposição de BISCHOF (1996, p. 680) sobre níveis agregados de ego figural vs. medial, podemos assinalar dois modos de identidade aos dois caminhos de socialização. A identidade figural ocidental emerge como uma construção cognitiva através de processos ativos de comparação social, enquanto que a estrutura de identidade não-ocidental consistiria predominantemente na adoção de um papel consignado. 


\section{PANORAMA}

Baseada em considerações evolucionárias concernentes à padronização do curso vital como parte de estilos reprodutivos de vida, tentamos identificar dois diferentes caminhos de socialização caractenzando: um estilo oci- dental para sociedades altamente isoladas (industrializadas), e um tipo não-ocidental que descreve principalmente padrões organizados de vida econômica por subsistência de contextos rurais de artesãos. Estes dois caminhos desenvolvimentais estão caracterizados no Quadro 3.

Quadro 3 - Caracterizando dois caminhos desenvolvimentais

\begin{tabular}{|c|c|c|}
\hline Fase da Recepção & \multicolumn{2}{|c|}{ Tarefa: Aquisição de uma matriz social } \\
\hline & não-Ocidental & Ocidental \\
\hline Mecanismos & $\begin{array}{l}\text { contato corporal extenso } \\
\text { extensa maternagem }\end{array}$ & $\begin{array}{l}\text { interação distal extensa } \\
\text { ritmo diurno precoce }\end{array}$ \\
\hline Resultados & $\begin{array}{l}\text { regulação-pelo-outro } \\
\text { relação mãe-criança simbiótica }\end{array}$ & $\begin{array}{l}\text { regulação - pelo-self precoce } \\
\text { relaçào mãe-criança } \\
\text { descentrada }\end{array}$ \\
\hline Conseqüência & $\begin{array}{l}\text { Self como Co-Agência } \\
\text { (relacional) }\end{array}$ & Self como Agência \\
\hline Fase de aquisição & \multicolumn{2}{|c|}{ Tarefa: Aquisição de competência } \\
\hline Mecanismos & $\begin{array}{l}\text { aprendizagem auto-responsável, cultura } \\
\text { como conteúdo da aprendizagem }\end{array}$ & $\begin{array}{l}\text { aprendizagem outrém-responsável } \\
\text { através de instrução; aprendizagem } \\
\text { de técnicas culturais }\end{array}$ \\
\hline Resultados & $\begin{array}{l}\text { conhecimento compartilhado, inteligência } \\
\text { como harmonia social; contribuição } \\
\text { precoce à renda familiar }\end{array}$ & $\begin{array}{l}\text { conhecimento privado. inteligência } \\
\text { lógico-formal, longa dependência } \\
\text { econômica }\end{array}$ \\
\hline Conseqüência & identidade como fundo & identidade como figura \\
\hline Fase de transformação & \multicolumn{2}{|c|}{ Tarefa: Aquisição de auto-conceitos } \\
\hline Mecanismos & $\begin{array}{l}\text { Co-construção de normas e valores } \\
\text { culturais tradicionais }\end{array}$ & $\begin{array}{l}\text { definição individual de normas e } \\
\text { valores tradicionais }\end{array}$ \\
\hline Resultados & formação familiar precoce & moratória educacional \\
\hline Conseqüência & Self como identidade grupo-relacionada & Self como identidade individual \\
\hline
\end{tabular}

In KELLER \& ECKENSBERGER (1998, p. 87).

Estes dois caminhos são compreendidos como dois modos comportamentais em que ambos pertencem ao repertório universal de abarcar disposições humanas comportamentais. Segundo as demandas contextuais, diferentes combinações destas propensões desenvolvimentais podem vir a ser observadas. Para poder qualificar estes caminhos desenvolvimentais como preparação para diferentes estilos reprodutivos, tem de ser analisado como os padrões reprodutivos diferem através do âmbito cultural aqui capturado. Como uma aproximação exploratória a esta questão, podemos comparar o número médio de crianças per mulheres e a média de expectativa de vida em anos. Nas sociedades ocidentais cerca de 1,7 crianças per mulher com uma expectativa de vida de 74,4 em média são relatados (BIRG, 1996; Demographic Yearbook of the United Nations, 1995), com a Itália tendo o número médio mais baixo de crianças $(1 ., 17)$ e uma das expectativas de vida mais altas $(78,8)$. Em 
sociedades não-ocidentais, de outro lado, o número médio de crianças é 3.5 com a expectativa média de vida de 62.3 (BIRG, 1996). DZEAYE (in prep.) relatou dados para dez tribos dos Camarões com um número médio de crianças de 5.2 com a expectativa média de vida de 50.2 anos. Estes dados podem of erecer algumas primeiras sugestões para a análise de diferentes práticas culturais de socialização como relacionadas a diferentes estilos de reprodução. A concepção sociobiológica de investimento parental pode, assim, ser estendida a modos parentais diferentes, refletindo diferentes qualidades antes do que variação quantitativa. As muitas questões abertas deveriam estimular a pesquisa empírica, particularmente estudos inter-culturais.

\begin{abstract}
The differentiation of the human life course into phases can be regarded as an adaptation to contextual demands. Especially sociobiological approaches have attributed developmental phases the status of phylogenetic achievements rather than transitory stages. Accordingly, pattern and duration of phases differ across contexts and cultures. Infancy is regarded as the phase where a primary social matrix is constructed. Although this developmental task is universal, the specific elaboration differs with different cultural backgrounds. The developmental results of this phase lay ground for specific ways of exploration and learning during childhood as the acquisition phase. A typical Western and a typical non-Western developmental trajectory are presented, which then lead to different conceptions of adolescence as an educational and self developing moratorium on the one hand and onset of the adult reproductive life on the other.
\end{abstract}

Key-words: life history research; socialization contexts; individualism.

\section{REFERÊNCIAS BIBLIOGRÁFICAS}

ANANDALAKSHMY, S. Socialization for competence. In: BERRY, J.W.; LONNER, J. (eds.). Applied cross-cultural psychology. Lisse, Swets \& Zeitlinger, 1975. p. 202-205.

ANDALAKSHMY, S. The female child in a family setting. The Indian Journal of Social Work, L11: 29-36, 1991.

BANDURA, A.; HUSTON, A.C. Identification as a process of incidental learning. Journal of Abnormal \& Social Psychology, 63: 31 1-3 18, 1961.

BAYLEY, N. On the growth of intelligence. American Psychologist, 10: 805-8 18, 1955.

BELSKY, J., STEINBERG, L.; DRAPER, P. Childhood experience, interpersonal development, and reproductive strategy: An evolutionary theory of socialization. Child Development, 62(4): 682-685, 1991.

BERRY, J. W.; BENNETT, J. A. Cree conceptions of cognitive competence. International Journal of Psychology: 27: 73-88, 1992.

BIRG, H. Die Weltbevolkerung. Dynamik und Gef ahren. München, Beck, 1996.

BISCHOF, N. Das Kraftfeld der Mythen. Signale aus der Zeit, in der wir die. Welt erschaffen haben. München,Piper, 1996.

CHASIOTIS, A. Zur intergenerationellen Bedeutung des Kindheitskontextes fur die somatische, psychologische und reproduktive Individualentwicklung. [Dissertation, Universitat Osnabruck, 1998].
CHISHOLM, J. Death, hope, and sex: Life-history theory and the development of reproductive strategies. Current Anthropology, 34(1) 1-24, 1993.

COLE, C. Culture in development. In: BORNSTEIN, M. H.; LAMB, M. E. (ed.). Developmental psychology. An advanced textbook. $3^{\text {rd }}$ ed. Hillsdale, N.J., Erlbaum, 1992.

DASEN, P.R.; DEMBELE, B.; ETTEN, K.; KABRAN, K.; KAMAGATE, D.; KAFFI, D.A.; N'GUESSAN, A. N'glouele, I'intelligence chez les Baoulé. Archives de Psychologie, 53: 293-324,1985.

DZEAYE, R.J. An investigation of breastfeeding and mother-infant interactions in the face of cultural taboos and belief systems. The case of Nso and Fulani mothers and their infants of 3-5 months of age in Mbvem, Sub-division of the North-west province of Cameroon. Manuskript, Universitat Osnabrück. (in prep.).

EIBL-EIBESFELDT, I. DieBiologie des menschlichen Verhaltens. Grundrift der Humanethologie. München, Piper, 1984.

El-SHAMY, H. The brother sister syndrome in Arab family life, sociocultural factors in Arab psychiatry: A critical review. International Journal of Sociology of the Family, 11: 313323,1981.

EMDE, R. N. The affective self: continuities and transformations from infancy. In: CALL, J.D.; GALENSON, E.; TYSON, RL. (eds.). Frontiers of infant psychiatry. New York, Basic Books,1984. p.38-54. 
GREENFIELD, P.M. Culture as process: empirical methods forcultulalpsychology. $\sim$ n: BERRY, J.W.; POORTINGA, Y. H.; PANDEY, J. (eds.). Handbookof cross cultural psychology. Vol. 1. Theory and method. 2. ed. Boston, Allyn \& Bacon, 1996. p.301-346.

GREENFIELD, P.M.; CHILDS, C. Weaving, colorterms, and pattern representation: cultural influences and cognitive development among the Zinacantecos of Southern Mexico. Inter-American Journal of Psychology, 11: 2348, 1977.

GREENFIELD, P.M.; CHILDS, C.P. Developmental continuity in biocultural context. In: COHEN, R.; SIEGEL, A.W. (eds.). Context and development. Hillsdale, NJ, Erlbaum, 1991. p. 135-159.

HARKNESS, S.; SUPER, C.M. Why African children are so hard to test. In: ADLER, L. L. (ed.). Crosscultural research at issue. New York, Academic Press,1977. p.145- 152.

HARLOW, H. F.; HARLOW, M.K. Social deprivation in monkeys. Scientific American, 207: 136-146, 1962.

HEWLETT, B. S. Intimate fathers: the nature and context of Aka Pygmy paternal infant care. Ann Arbor, University of Michigan Press, 1991.

JOLLY, A. Die Entwicklung des Primatenverhaltens. Stuttgart, Fischer, 1975.

KAGITCIBACI, C. Individualism and collectivism. In: BERRY, J. W.; SEGALL, M. H.; KAGITCIBASI, C. (eds.). Handbook of CrossCultural psychology. Volume 3. Social Behavior and applications. 2. ed. Boston, Allyn \& Bacon, 1997. p.1-49.

KELLER, H. Evolutionary approaches to the life span. In: GELEN, U.P.; COMUNIAN, A. L. (eds.). Crosscultural perspectives on human development. Padua, Cedam. (1998 in press).

KELLER, H.; LOHAUS, A.; VÖLKER, S.; CAPPENBERG, M.; CHASIOTIS, A. Temporal contingency as a independent component of parenting behavior. (in prep. a)

KELLER, H. \& ECKENSBERGER, L. H. Kultur und Entwicklung. In: Keller, H. (ed.). Lehrbuch Entwicklungspsychologie. Bern, Huber Verlag, 1998. p. 57-96.

KELLER, H.; CHASIOTIS, A.; ZACH, U.; VOELKER, S.; LOHAUS, A. An evolutionary account of parenting. (in prep. b).

KELLER, H.; VÕLKER, S.; ZACH, U. Attachment in cultural context. Newsletter ISSBD, 1 (31): 1-3, 1997.
LeVINE, R.A. Infant environments in psychoanalysis. A cross-cultural view. In: STIGLER, J. W., SHWEDER, R.A.; HERDT, G. (eds.). Cultural psychology. Essays on comparative human development. Cambridge, Cambridge Universitv Press,1990. p.454-474.

LLOBERA, J. R. Naturvolker. Sozialsysteme im Einklang mit der Umwelt. Reinbek/Hamburg, Rowohlt, 1978.

MacDONALD, K.B. Social and personality development. An evolutionary synthesis. NY, PlenumPress, 1988.

MacDONALD, K.B. Evolution, culture, and the fivefactor model. Journal of Cross-Cultural Psychology, 29(1): 119-149, 1998.

MARKUS, H.; KITAYAMA, S. Culture and the self implications for cognition, emotion and motivation. Psychological Review, 98: 224253, 1991.

MARKUS, H.; KITAYAMA, S. The cultural psychology of personality. Journal of CrossCultural Psychology, 29 (1): 63-87, 1998.

MUNROE, R. H.; MUNROE, R. L. Behavior across cultures: Results from observational studies. In: LONNER, W.J.; MALPASS, R.S. (eds.). Psychology and culture. Boston, Allyn \& Bacon,1994. p.107- 111.

MVENG, E. Initiation ordinaire et inihation charismatique. Anthropos, 2: 16-25, 1974. (2éme séminaire anthropologique du Grand Séminaire de Nkol-Bisson,25 Feb. - 1 Mars)

MZEKA, P. N. Rituals of initiation in the Western grassfields of Cameroon: The NSO case. Reprinted from: Rites of Passage and incorporation in the West grassfields of Cameroon. Vol. 1: From birth to adolescence. Bamenda, Cameroon, The Kaberry Research Centre, 1996.

NSAMENANG, A.B. Human deve^lopment in cultural context. A third world perspective. Newbury Park, Sage,1992.

PIAGET, J. La naisssance de l'intelligence chez l'enfant. Paris, RU.F,1939.

PIANKA, E. R. On r- and K-selection. American Naturalist, 104: 592-597,1970.

RABAIN-JAMAIN, J. L'enfant du liguage. Du sevrage à la classe d'âge chez les Wolof du Sénégal. Paris, Payot,1979.

RABINOVICH, E.P. Comparative study of sleeping arrangements and breastfeeding in Brazilian children. Fourteenth International Association For Cross-Cultural Psychology Congress - 1998, and Silver Jubilee Congress. Bellingham Washington, IACCP,1998. p.95.

ROGOFF, B. Apprenticeship in thinking. cognitive development in social context. New York, Oxford University Press, 1990. 
ROGOFF, B.; MISTRY, J.; GONCU, A.; MOSIER, C. Guided participation in cultural activity by toddlers and caregivers. Monographs of the Society for Research in Child Development, Serial No. 236, 58(8),1993.

RUDMIN, F. W. Will the real Euro-centrists please stand up? Bulletin of Cross-Cultural Psychology, 30(4): 4-5, 1996.

SARASWATHI, T. S. Women in poverty context: balancing economic and child care needs. In: BOROOAH, R.; CLOUD, K; SESHADRI, S.; SARASWATHI, T.S.; PETERSON, J T.; VERMA, A. (eds.). Capturing complexity. An interdisciplinary look at women, households and development. New Dehli, Sage, 1994. p. 162-178.

SARASWATHI, T. S.; PAI, S. Socialization in the Indian context. In: KAO, H. S. R.; SINHA, D. (eds.). Asian perspectives on psvchology. New Dehli, Sage, 1997. p. 74-92.

SEGINER, R. Hopes and fears of anticipated adulthood. Adolescents' future orientation in cross-cultural context. In: TROMMSDOREF, G. (ed.). Kindheit und Jugend in verschiedenen Kulturen. Weinheim/München, Juventa, 1995. p. 225-247.

SERPELL, R. 'Estimates of intelligence in a rural community of Eastem Zambia'. In: OKATCHA, F. M. (ed.) Modern p.sychology and cultural adaptation. Nairobi, Swahili Language Consultants and Publishers, 1977. p. 1 79216.

SERPELL, R. Research on cognitive development in sub-Saharan Africa. International Journal of Behavioral Development, 7: 111-127, 1984.

SHOSTAK, M. Nisa. The life and words of a !Kung woman. Carnbridge, Harvard University Press, 1981.

SHWEDER, R.A.; BOURNE, R. A. Does the concept of a person vary cross-culturally? In: MARSELLA. A.J.; WHITE, G. M. (eds.). Cultural conceptions of inental health and therapy. London, Reidel,1982. p.97-137.
SROUFE, A.; WATERS, E. On the universality of the link between responsive care and secure base behavior. ISSBD-Newsletter, 31(1): 3-5, 1997.

SUPER, C.; HARKNESS, S. The cultural structuring of child development. In: BERRY, J. W.; DASEN, P. R.; SARASWATHI, T.S. (eds.). Handbook of Cross-cultural psychology. Vol. 2. Basic processes and human development. 2. ed. Boston, Allyn \& Bacon, p.1-39, 1996.

TARABULSY, G. M.; TESSIER, R.; KAPPAS, A. Contingency detection and the contingent organization of behavior in interactions: Implications for socioemotional development in infancy. Psychological Bulletin, 120: 25-41, 1996.

TRONICK, E. Z.; MORELLI, G. A.; IVEY, P. K. The Efe forager infant and toddler's pattern of social relationships: Multiple and simultaneous. Developmental Psychology, 28(4): 563-577, 1992.

Van GENNEP, A. The rites of passage. In: VIZEDON, M. B.; CAFFEE, G. L. (eds.). Chicago, University of Chicago Press, 1960.

VÖLKER, S.; KELLER, H.; LOHAUS, A.; CAPPENBERG, M;. CHASIOTIS, A. Maternal interactive behavior in early infancy and later attachment. International Journal of Behavioral Development. (in press)

WALDEN, T. A. Social responsivity: judging signals of young children with and without developmental delays. Child Development, 67(5): 2074-2085, 1996.

WHITING, J. W. M. Environmental constraints on infant care practices. In: MUNROE, R. H.; MUNROE, R. L.; WHITING, B. B. (eds.). Handhook of cross-cultural human development. New York, Garland, 1981. p. 155-179.

WHITING, J. W. M. Adolescent rituals and identity conflicts. In: STIGLER, J. W.; SHWEDER, R.A.; HERDT, G. (eds.). Cultural psychology, Essays on comparative human development. New York, Carnbridge University Press,1990. p.357-365. 